Journal of

Molecular Microbiology

and Biotechnology
DOI: $\underline{10.1159 / 000345769}$

Published online: December 28, 2012

\title{
Prediction, Microarray and Northern Blot Analyses Identify New Intergenic Small RNAs in Aliivibrio salmonicida
}

\author{
Rafi Ahmad Geir Åsmund Hansen Hilde Hansen Erik Hjerde \\ Hege Lynum Pedersen Steinar M. Paulsen May Liss Julianne Nyrud \\ Anja Strauss Nils-Peder Willassen Peik Haugen
}

The Norwegian Structural Biology Centre and Department of Chemistry, Faculty of Science and Technology, University of Tromsø, Tromsø, Norway

\section{Key Words}

Small RNA • Microarray • Non-coding RNA • Vibrionaceae

\section{Abstract}

Bacterial small RNAs (sRNAs) are trans-encoded regulatory RNAs that typically bind mRNAs by short-sequence complementarities and change the expression of the corresponding proteins. Some of the well-characterized sRNAs serve critical steps in the regulation of important cellular processes, such as quorum sensing (Qrr), iron homeostasis (RyhB), oxidative stress (OxyS), and carbon metabolism (Spot 42). However, many sRNAs remain to be identified, and the functional roles of sRNAs are known for only a small fraction. For example, of the hundreds of candidate sRNAs from members of the bacterial family Vibrionaceae, the function is known for only 9. We have in this study significantly contributed to the discovery and verification of new sRNAs in a representative of Vibrionaceae, i.e. the Aliivibrio salmonicida, which causes severe disease in farmed Atlantic salmon and other fishes. A computational search for intergenic non-coding (nc) RNAs in the 4.6-Mb genome identified a total of 252 potential ncRNAs (including 233 putative sRNAs). Depending on the set thresh- old value for fluorescence signal in our microarray approach, we identified 50-80 putative ncRNAs, 12 of which were verified by Northern blot analysis. In total, we identified 9 new sRNAs.

Copyright $\odot 2012$ S. Karger AG, Basel

\section{Introduction}

Bacteria contain a diverse set of non-coding (nc) RNAs [Westhof, 2010]. Some of these, such as RNaseP, tRNAs, rRNAs, and tmRNA, serve 'housekeeping' functions, CRISPR RNAs protect the bacteria from viruses and plasmids [Horvath and Barrangou, 2010], whereas other classes of ncRNAs serve as regulators in gene expression. cis-encoded RNA regulators (i.e. riboswitches) are typically part of mRNAs and located in front of protein coding regions and change the expression of the corresponding protein by binding to small metabolites [Breaker, 2011]. Other regulatory RNAs are transcribed in trans from separate promoters located in intergenic regions (IGRs), or from the opposite strand (i.e. anti-sense RNAs) of protein coding regions.

\section{KARGER}

Fax +4161306 1234

E-Mail karger@karger.ch

www.karger.com
(C) 2012 S. Karger AG, Basel

$1464-1801 / 12 / 0226-0352 \$ 38.00 / 0$

Accessible online at:

www.karger.com $/ \mathrm{mmb}$
Peik Haugen

The Norwegian Structural Biology Centre and Department of Chemistry

Faculty of Science and Technology, University of Tromsø

NO-9037 Tromsø (Norway)

E-Mail peik.haugen@uit.no 
The majority of trans-encoded regulatory RNAs in bacteria are small in size (hence the name small RNAs or sRNAs) and act by targeting mRNAs by short imperfect sequence complementarities, thus modulating expression of their targets [Gottesman, 2005]. The result can be change in cellular processes, such as iron homeostasis [Ahmad et al., 2009; Masse and Gottesman, 2002], quorum sensing [Hammer and Bassler, 2007], sugar metabolism [Vanderpool, 2007], oxidative stress [Altuvia et al., 1997], and virulence [Romby et al., 2006]. sRNAs were first discovered in Escherichia coli 30 years ago [Griffin, 1971; Ikemura and Dahlberg, 1973], but their abundance and important functional roles have only recently been acknowledged. By the end of the 20th century, only 10 sRNA were known in E. coli [Wassarman et al., 1999], but this quickly changed when several research labs started genome-wide systematic searches [Argaman et al., 2001; Chen et al., 2002; Rivas and Eddy, 2001; Wassarman et al., 2001]. After these initial studies in E. coli, a number of similar works have resulted in a growing list of sRNAs or putative sRNAs from other bacteria (mostly pathogens), e.g. Bacillus subtilis [Irnov et al., 2010], Vibrio cholerae [Liu et al., 2009; Livny et al., 2005], Pseudomonas aeruginosa [Livny et al., 2006], Staphylococcus aureus [Pichon and Felden, 2005], and Salmonella typhimurium [Ortega et al., 2012]. As of May 2012, the sRNAMap database lists 79 sRNAs in E. coli strain K12 MG1655 (87 in all E. coli strains) and a total of 397 in 28 bacterial species [Huang et al., 2009]. Several approaches, such as bioinformatic prediction, microarray, RNA-seq, Hfq coimmunoprecipitation, and RNomics, have proven useful in genomewide detection of bacterial sRNAs [Sharma and Vogel, 2009], and we expect that a significant number of sRNAs will be discovered in future studies.

The relatively large Vibrionaceae family of gammaproteobacteria contains a number of serious pathogens of humans (e.g. V. cholerae, Vibrio parahaemolyticus, and Vibrio vulnificus) and animals (e.g., Vibrio coralliilyticus, Vibrio anguillarum, and Alivibrio salmonicida) [reviewed in Thompson et al., 2004]. These bacteria are therefore of broad interest, and several recent efforts have focused on genome-wide discovery of sRNAs in representatives of Vibrionaceae, mostly using V. cholerae as the model [Liu et al., 2009; Livny et al., 2005]. Together, these studies identified hundreds of putative sRNAs and verified the presence of 13 by Northern blot analysis. Experimental evidence of specific roles of sRNA in vibrios and aliivibrios (bacteria from the two genera Vibrio and Aliivibrio, respectively) is known for Qrr1-5 and the redundant $\mathrm{CsrB}$, CsrC, and $\mathrm{CrsD}$, which all regulate quorum sensing [Lenz et al., 2005, 2004], RyhB, which is involved in iron homeostasis [Davis et al., 2005], MicX [Davis and Waldor, 2007] and VrrA [Song et al., 2008], which regulates expression of outer membrane proteins, MtlS (previously designated the IGR7 sRNA), which inhibits the synthesis of the mannitol-specific phosphotransferase system [Mustachio et al., 2012], and MRB RNA I, which regulates plasmid replication [Le Roux et al., 2011]. Recently, we presented a study on A. salmonicida, in which new roles for Spot 42 sRNA and the discovery of the neighboring VSsrna24 sRNA were presented [Hansen et al., 2012]. Spot 42 appears to have a central role in the central metabolism by regulating the crucial step catalyzed by pyruvate dehydrogenase.

We have in this study performed a genome-wide computational search for ncRNAs in IGRs with special focus on sRNAs in the genome of the Gram-negative and coldadapted $A$. salmonicida. The bacterium is the causative agent of cold-water vibriosis (or Hitra disease) in farmed Atlantic salmon, sea farmed rainbow trout, and captive Atlantic cod. Computational predictions were used for custom design of a microarray chip containing ncRNA predictions, as well as nearly all open reading frames. Twenty-eight ncRNAs, including 26 previously unrecognized sRNAs, were tested by Northern blot analysis.

\section{Materials and Methods}

Bacterial Strains and Growth Conditions

A. salmonicida LFI1238 [Hjerde et al., 2008] and an isogenic $\Delta$ litR mutant [Bjelland et al., 2012] were cultured in LB medium containing $2.5 \% \mathrm{NaCl}$ at $12{ }^{\circ} \mathrm{C}$ and $200 \mathrm{rpm}$. In three independent experiments (biological replicates), cells were subjected to $\mathrm{H}_{2} \mathrm{O}_{2}$ or 2,2'-dipyridyl after growth in 200 -ml culture flasks to midexponential phase $\left(\mathrm{OD}_{600} \sim 0.5\right)$. Cells were next split into three equally sized cultures. $\mathrm{H}_{2} \mathrm{O}_{2}$ was added to one flask to a final concentration of $100 \mu \mathrm{M}$, the iron chelator 2,2'-dipyridyl was added to one flask to a final concentration of $50 \mu \mathrm{M}$, and, finally, one flask was used as the non-treated control. Two 5-ml samples were collected 7.5, 15, and $30 \mathrm{~min}$ after treatment, and cells were harvested by centrifugation $(3,500 \mathrm{~g}, 10 \mathrm{~min})$, flash frozen, and kept at $-70^{\circ} \mathrm{C}$ prior to RNA isolation. The A. salmonicida $\Delta$ litR mutant was used to find sRNAs with potential roles in quorum sensing. In three independent experiments (biological replicates), the $\Delta$ litR mutant was grown in 60-ml cultures and samples were collected at $\mathrm{OD}_{600 \mathrm{~nm}} 0.15,0.5$, and 0.8 for microarray analysis, and $\mathrm{OD}_{600 \mathrm{~nm}} 0.15,0.5,0.8$, and 1.2 for Northern blotting. Wild-type strain was used as control. Samples were subsequently treated as described above.

\section{Northern Blot Analyses}

Total RNA was isolated from bacterial cultures using isolRNA reagent (5 PRIME) and quantified with NanoDrop (Thermo Fisher Scientific). Approximately $10 \mu \mathrm{g}$ of total RNA was sepa- 
rated on $5 \%$ polyacrylamide/8 $\mathrm{M}$ urea denaturing gels and transferred to a positively charged Hybond-N+ nylon membrane (GE Healthcare) by a TE70 series SemiPhor Semi-Dry Transfer Unit (Hoefer Inc.). Subsequent procedures were done essentially as previously described [Ahmad et al., 2009]. Briefly, RNAs were detected on membranes by using $\left[\alpha{ }^{32} \mathrm{P}\right]$ dCTP-labeled doublestranded DNA probes, and signals were collected on phosphoimaging screens (Fujifilm) and scanned on a BAS-5000 phosphoimager (Fujifilm). The ImageGauge software v4.0 (Fujifilm) was used to measure the strength of signals. $5 \mathrm{~S}$ ribosomal RNA was used to normalize the resulting values.

\section{Microarray Analyses}

Microarray analysis was run as described in Pedersen et al. [2010]. Briefly, RNA preparations from three independent experiments (biological replicates) were subjected to DNase treatment (to remove DNA) and a clean-up step using RNeasy MinElute spin columns (Qiagen). The three independent RNA samples were then pooled before cDNA synthesis. The quality of RNA was tested, and cDNA was constructed from $15 \mu \mathrm{g}$ purified RNA using the Aminoallyl cDNA Labeling Kit (Ambion) and labeled using CyDye ${ }^{\mathrm{TM}}$ Post-Labeling Reactive Dye Pack (GE Healthcare). Labeled samples were hybridized to three 'Vibrio salmonicida V1.0.1 AROS' slides (Eurofins; technical replicates), and slides were subsequently washed. Slides were finally scanned, and resulting expression data were analyzed using J-Express Pro v2.7 [Dysvik and Jonassen, 2001]. Microarray data have been uploaded to The NCBI Gene Expression Omnibus database and are available through accession number GSE25558. Expression data from $\mathrm{H}_{2} \mathrm{O}_{2}$-treated cells were extracted from GSE20082 [Pedersen et al., 2010].

\section{Computational Prediction of sRNAs}

The search for potential sRNA genes in A. salmonicida was limited to IGRs. We define an IGR as the region between two annotated genes on either strand that is delimited by its closest left and right flanking genes. Briefly, our sRNA genes were identified by searching for co-localization of genetic features that are associated with bacterial sRNA genes. These include (1) the presence of putative promoters and/or transcription factor binding sites 50$500 \mathrm{nt}$ upstream of Rho-independent terminators, (2) sequence conservation between phylogenetically related species, and (3) conservation of synteny of flanking genes.

BProm (Softberry, Mount Kisco, N.Y., USA; http://www.softberry.com), a bacterial $\sigma^{70}$ promoter recognition program, was used to predict -10 and -35 promoter sequences. Specific positionspecific scoring matrices for $\sigma^{54}$ and $\sigma^{38}$ were used as input to Patser [Hertz and Stormo, 1999] to scan the A. salmonicida IGRs for $\sigma^{54}$ and $\sigma^{38}$ promoter sequences. Prediction of potential Furbinding sites was done as previously described [Ahmad et al., 2009]. TransTerm [Ermolaeva et al., 2000] was used to predict Rho-independent terminators in both chromosomes of $A$. salmonicida. TransTerm searches were conducted with the confidence threshold of $90 \%$. The genome viewer and annotation tool Artemis [Rutherford et al., 2000] and a python script was used to visualize the predicted sequence features described above. The ncRNA prediction programs sRNAfinder [Tjaden, 2008] and QRNA [Rivas and Eddy, 2001] were run for comparison and validation of our predictions.
Compilation of a Vibrionaceae IGR Database and Search for sRNA Homologs

The IGRs from A. salmonicida were used to search for homologous IGRs in the six Vibrionaceae genomes of $V$. cholerae O1 biovar eltor str. N16961, V. fischeri ES114, V. parahaemolyticus RIMD 2210633, V. vulnificus CMCP6, V. vulnificus YJ016, and Photobacterium profundum SS9. Sequences were extracted from GenBank (ftp://ftp.ncbi.nlm.nih.gov/genbank/genomes/Bacte$\mathrm{ria} /$ ). An IGR database was constructed based on these six genomes and gene annotation as provided at NCBI's Complete Microbial Genomes (http://www.ncbi.nlm.nih.gov/genomes/lproks. cgi). Only IGRs $\geq 50$ nt in length were used. The Formatdb option was used to format the IGR database. StandAlone BLAST from NCBI (ftp://ftp.ncbi.nih.gov/blast/) was used to search for homologs of putative sRNAs. Blastn search parameters were set to word size $=7$ and $\mathrm{E}$-value $\leq 0.1$.

The search for homologs of known ncRNAs was performed using the cmsearch program, which is part of the Infernal software package [Nawrocki et al., 2009] and the Rfam database of ncRNA families [Gardner et al., 2009]. The lower cmsearch cutoff score was set to 25 . Sequences corresponding to tRNAs and rRNAs were excluded.

\section{Results and Discussion}

\section{A. salmonicida Total RNA Is Rich in RNAs of \\ 150-200 nt}

To evaluate the potential for finding new sRNAs in $A$. salmonicida, we initially cultivated the bacterium and sampled cells at early, mid-, and late exponential phase (i.e. $\mathrm{OD}_{600 \mathrm{~nm}} 0.15,0.75$, and 1.0). Total RNA from these samples was separated on a $5 \%$ polyacrylamide $/ 8 \mathrm{M}$ urea gel (fig. 1). Major bands were identified as 23S, 16S, and $5 \mathrm{~S}$ rRNAs and multiple tRNAs based on predicted sizes and previous publications [e.g. Pichon and Felden, 2005]. Interestingly, a number of faint but distinct bands in the size range of $150-200 \mathrm{nt}$ are clearly visible. The size range overlaps well with the expected sizes of bacterial sRNAs [Huang et al., 2009]. To evaluate if at least a fraction of these bands could correspond to sRNAs or other types of ncRNAs, we isolated RNAs of 150-300 nt in length from the gel, and cloned the corresponding cDNA in a shotgun approach. Sanger sequencing of 110 clones revealed that the majority of these corresponded to rRNA (60\%) or mRNA (25\%) fragments, and only five clones mapped to IGRs. In summary, our initial survey showed that A. salmonicida total RNA contains numerous sRNA species. However, the majority of these correspond to rRNA or mRNA fragments, and only a very limited number $(\sim 4 \%)$ map to IGSs (i.e. potential sRNAs). 
Bioinformatic Prediction of 252 Putative ncRNAs

The experimental data described above provided us with limited information about the potential for finding sRNAs, so in a parallel approach, we used bioinformatics to predict ncRNA genes in IGRs. The A. salmonicida genome consists of two chromosomes and four plasmids [Hjerde et al., 2008], and we extracted a total of 3,308 IGRs constituting $23 \%$ of the genome. We considered only IGRs $\geq 50 \mathrm{nt}$ in length, which delimited the number of IGRs to 2,488 (approx. 21\% of total genome). The majority of these $(1,828)$ map to chromosome I.

A search in Rfam identified 25 homologs of known ncRNAs (online suppl. table $\mathrm{S} 1$ with detailed information on predicted ncRNAs from intergenic regions of chromosomes I and II in A. salmonicida; for all online suppl. material, see www.karger.com/doi/10.1159/000345769) distributed among the categories sRNAs, riboswitches, RNaseP, and tmRNA (excluding tRNAs and rRNAs). Next, putative sRNAs were predicted based on three main criteria: (1) colocalization of putative promoters and/or transcription factor binding sites and Rho-independent terminators (50-500 nt apart), (2) high sequence conservation between relatively closely related species, and (3) conservation of synteny of flanking genes. Figure 2 shows how the 252 predicted ncRNAs (196 in ChrI and 56 in ChrII) are distributed in size, ranging from 65 to $392 \mathrm{nt}$. The majority is between 101-150 nt and 151-200 nt (73 and 92 , respectively), and $87 \%$ are $\leq 250 \mathrm{nt}$, which is in agreement with the typical size of sRNAs ( 233 of 252 predicted ncRNAs are candidate sRNAs). Promoters were predicted upstream of 48 by BProm and Patser; Furboxes were predicted upstream of 14 , and 89 were found associated with Rho-independent terminators using TransTerm. Compared to other available bioinformatic sRNA prediction tools, 192 of the predicted sRNAs were recovered by either sRNAfinder or QRNA. Finally, 222 are also found in one or more members of the Vibrionaceae family.

\section{Global Validation of ncRNA Predictions Using Microarray}

Next, we wanted to validate our ncRNA predictions on a global scale and used a custom-designed microarray (Vibrio salmonicida V1.0.1 AROS) from Operon (now Eurofins) based on 70-mer oligos. The microarray contains oligos complementary to all the 252 predicted ncRNAs (and nearly all the 4,282 protein coding genes, which are not considered here). We assumed that only a fraction of sRNAs would be expressed under normal lab conditions, and we therefore subjected A. salmonicida to

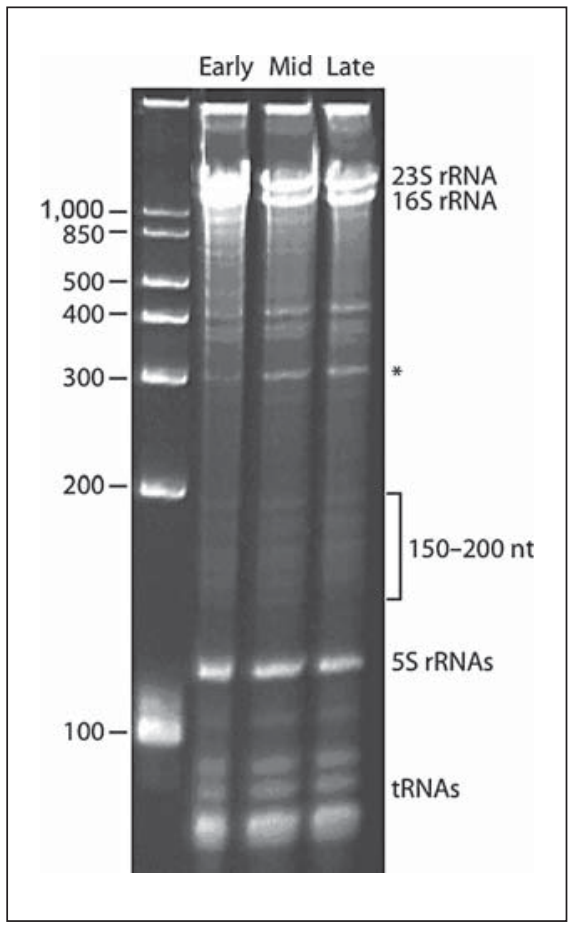

Fig. 1. A. salmonicida total RNA from early, mid- and, late exponential growth phase separated on a $5 \%$ denaturing polyacrylamide gel and stained with ethidium bromide. The positioning of $23 \mathrm{~S}, 16 \mathrm{~S}$, and $5 \mathrm{~S}$ rRNAs and tRNAs was inferred from the predicted sizes. A number of distinct bands can be seen in the size range of approximately 150-200 nt. The asterisk indicates a band of approximately $300 \mathrm{nt}$ that corresponds to a $23 \mathrm{~S}$ rRNA fragment that was identified by gel isolation, cDNA synthesis, and cloning.

low iron conditions and oxidative stress. Cells were grown to mid-exponential phase $\left(\mathrm{OD}_{600 \mathrm{~nm}} \sim 0.5\right)$, subjected to sub-lethal stress conditions by adding $50 \mu \mathrm{M} 2,2^{\prime}$ dipyridyl and $100 \mu \mathrm{M}$ fresh hydrogen peroxide, respectively, and samples were collected 15,30, and 60 min after the addition of chemicals. Treated samples were compared with untreated control samples. To find sRNAs with potential roles in quorum sensing, we also analyzed gene expression in a LitR deletion mutant $(\Delta l i t R)$ of $A$. salmonicida [Bjelland et al., 2012] (the $\Delta$ litR mutant will be described in detail elsewhere). LitR is the homolog of HapR from $V$. cholerae, which is the master regulator of quorum sensing [reviewed in Milton, 2006]. Here, samples were collected at low, mid, and high exponential growth phase $\left(\mathrm{OD}_{600 \mathrm{~nm}} 0.15,0.5\right.$, and 0.8 , respectively) and compared with identical samples of wild type.

Table 1 shows a summary of the microarray results (complete microarray datasets with fold change and fluo- 
Fig. 2. Size distribution of predicted ncRNAs. Predicted ncRNAs are shown in dark grey bars, ncRNAs that produced microarray fluorescence signal $>2$-fold above background are shown in light grey bars, and ncRNAs that produced microarray fluorescence signal $>100$ are shown in open bars.

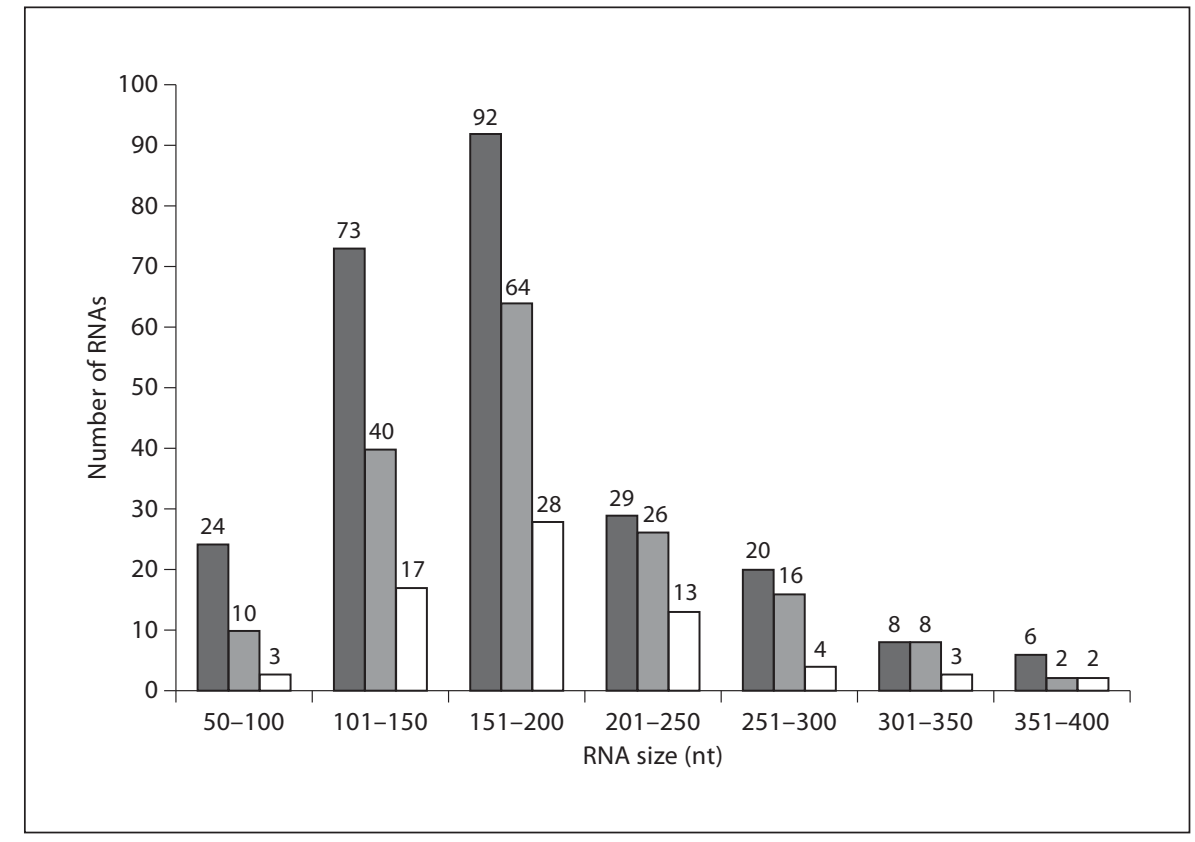

Table 1. Summary of ncRNA microarray analysis

\begin{tabular}{|c|c|c|c|c|c|c|}
\hline & \multirow[t]{2}{*}{ Total $^{1}$} & \multicolumn{2}{|c|}{ Fold change } & \multicolumn{3}{|c|}{ Threshold } \\
\hline & & $\geq 2$ & $\geq 1.5$ & $>50$ & $>75$ & $>100$ \\
\hline Low iron & 134 & 7 & 22 & 85 & 68 & 52 \\
\hline Oxidative stress & 142 & 4 & 26 & 91 & 62 & 50 \\
\hline$\Delta l i t R$ & 152 & 15 & 50 & 72 & 60 & 48 \\
\hline
\end{tabular}

${ }^{1}$ Total number of ncRNAs with fluorescence signal $2 \times$ above background.

rescence intensity values for each predicted ncRNA are available in online suppl. table S2), and figure 2 provides a graphical overview of how expressed ncRNAs are distributed into different size categories. Regardless of origin of samples (i.e. treated or untreated samples, or mutant or wild type), 134, 142, and 152 ncRNAs produced fluorescence signals $>2$-fold above background, and 7, 4, and 15 were differentially expressed under low iron, $\mathrm{H}_{2} \mathrm{O}_{2}$ conditions, or in $\Delta l i t R$, respectively. However, these numbers are likely inflated due to unspecific signals and/or false-positive predictions. A more conservative estimate would be to only consider spots with Cy 5 or Cy 3 fluorescence intensities above certain threshold values. We considered three different threshold values, i.e. $>50,>75$, and
$>100$. These were chosen based on the following observations and assumptions: Of the approximately $\sim 4,200$ genes that are spotted on the chip, about 3,600 were retained after bioinformatic analysis. With threshold values set at $>50,>75$, or $>100$, approximately $1,200,800$, or 700 genes are retained, respectively, which would suggest that at least $33(1,200 / 3,600), 22(800 / 3,600)$, or $19 \%$ $(700 / 3,600)$ of genes are expressed under our growth conditions when using microarray technology. These numbers are very modest when compared to recent RNA-seq data from for example Bacillus anthracis, which suggests expression of $\sim 94 \%$ of the entire genome [Passalacqua et al., 2009]. Even though it is difficult to directly compare data from these two very different technologies, the data indicate that our set threshold value of $>100$ is relatively conservative, because it is reasonable to believe that more than $19 \%$ of genes should be expressed during growth.

Table 1 shows that the average number of spots with fluorescence values above 50, 75, and 100 are 83, 63, and 50 , respectively. These spots represent putative sRNAs. Given that the total number of sRNAs in E. coli is 87, and that $A$. salmonicida has a similar number of sRNAs, it is reasonable to believe that many of the identified spots could represent genuine sRNAs.

Nine New sRNAs Detected by Northern Blot Analysis

Candidate sRNAs from table 1 were next tested by Northern blot analysis. The experimental setup was iden- 
Fig. 3. Northern blot analysis of selected ncRNAs. Radiolabeled double-stranded DNA probes that were specific for predicted sRNAs or one of the three control ncRNAs (Qrr, RNaseP, and 5S rRNA) were hybridized to membranes with total RNA from A. salmonicida wild type or $\Delta l i t R$ mutant. Prior to Northern blot analysis, wild-type cells were grown to mid-exponential phase and treated with $50 \mu \mathrm{M} 2,2^{\prime}$ dipyridyl or $100 \mu \mathrm{M}$ hydrogen peroxide. Untreated (control) cells were used as control. Samples were collected 7.5, 15, and 30 min after treatment. Numbers to the right represent actual and predicted RNA sizes. RNAs from chromosome I (Chr I) or II (Chr II) are indicated. The probe targeted against VSsrna46 produced multiple bands, and band sizes that did not correspond to the predicted size are shown in parentheses. 5S rRNA was used to normalize the strength of signals between lanes as measured with phosphoimaging. The VSsrna185 corresponds to the B2 sRNA that was recently identified in $V$. cholerae [Livny et al., 2005].

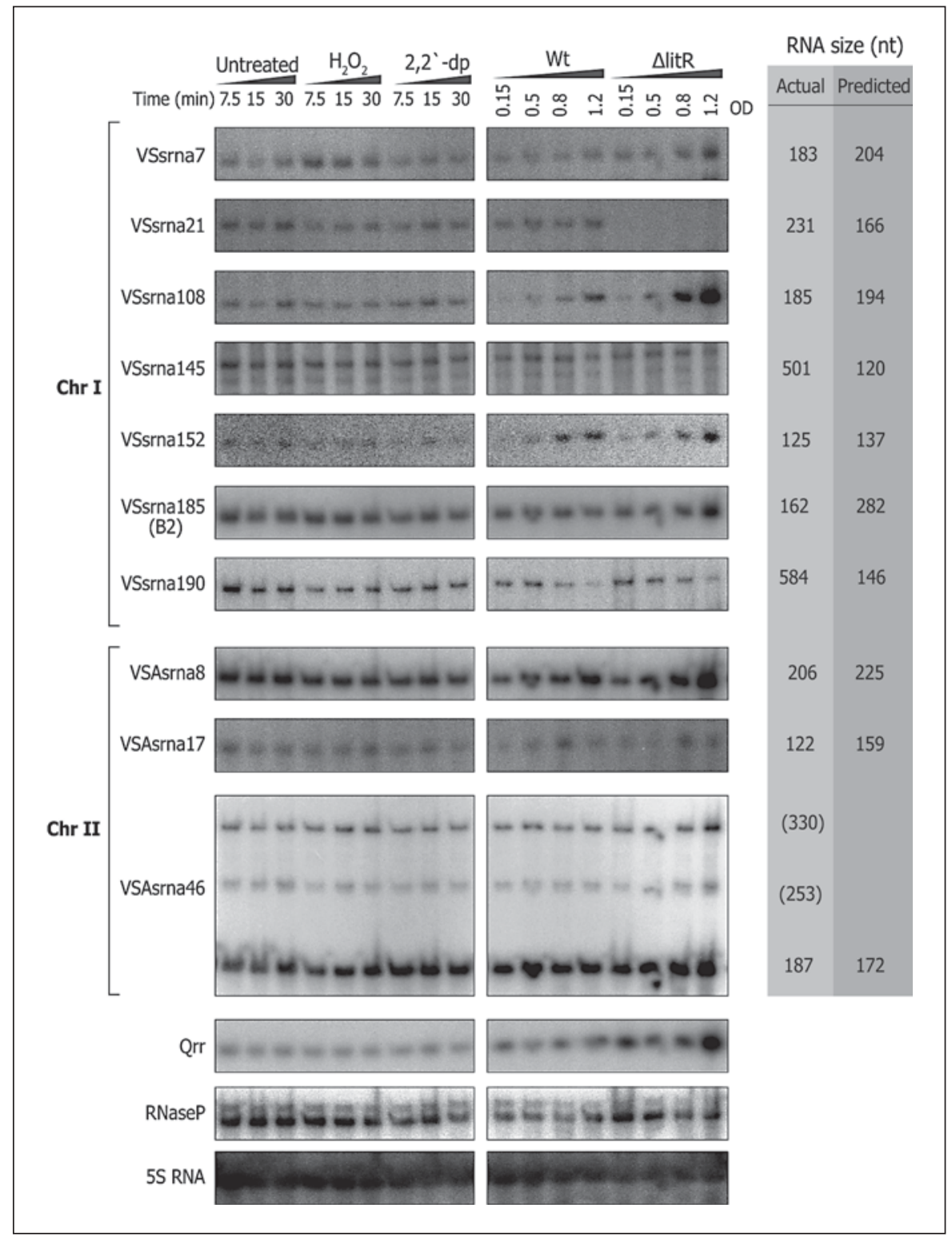

tical to that used for microarray, except that cell samples were collected 7.5, 15, and $30 \mathrm{~min}$ after treatment (instead of 15,30 , and $60 \mathrm{~min}$ ) or at ODs $0.15,0.5,0.8$, and 1.2 (OD 1.2 was added) for the $\Delta l i t R$ mutant. Probes were designed for 26 putative sRNAs. They were selected because they were highly and/or differentially expressed in the microarray experiment. The Qrr sRNA, RNaseP, and 5S rRNA were included as controls.

Figure 3 shows a summary of resulting Phosphoimage scans of 10 putative sRNAs (VSsrna 7, 21, 108, 145, 152, 185, and 190 on Chr I and VSAsrna 8, 17, and 46 on Chr II) and the three controls. Table 2 shows more details on these validated RNAs. VSsrna185 is a homolog of the B2 sRNA that was found in V. cholerae [Livny et al., 2005]. Predicted and actual RNA sizes are indicated. Thirteen probes did not produce a visible signal, and probes for VSsrna 10, 42, and 115 on Chr I produced multiple bands that could not be resolved and are not shown. As expected, Qrr was differentially expressed in the $\Delta$ litR mutant, from 2- to 5.5-fold upregulation (compared to wild type), which is in agreement with the microarray results (3.4to 5.6-fold upregulation). In V. cholerae, the gene expression levels of Qrr are fine-tuned by two feedback loops that involve HapR (i.e., the LitR homolog) and LuxO, 
Table 2. Details of experimentally validated sRNAs

\begin{tabular}{|c|c|c|c|c|c|c|c|c|c|}
\hline ncRNA ID & Flanking genes ${ }^{1}$ & $\operatorname{Start}^{2}$ & End $^{2}$ & Length ${ }^{3}$ & Strand $^{4}$ & $\begin{array}{l}\text { Sequence } \\
\text { conservation }^{5}\end{array}$ & Promoter $^{6}$ & $\begin{array}{l}\text { Termi- } \\
\text { nator }^{7}\end{array}$ & Type $^{8}$ \\
\hline VSsrna7 & VSAL_I0041/VSAL_I0042 & 44694 & 44491 & 204 & $<<>$ & + & - & - & Novel \\
\hline VSsrna21 & VSAL_I0222/VSAL_I0223 & 267228 & 267063 & 166 & $><<$ & +++ & SigmaS, Fur-box & + & Novel \\
\hline VSsrna108 & VSAL_I1796/VSAL_I1797 & 1919211 & 1919404 & 194 & $>>>$ & + & Fur-box & + & Novel \\
\hline VSsrna114 & VSAL_I1873/VSAL_I1874 & 2009822 & 2009717 & 106 & $><>$ & ++ & Sigma54 & + & Qrr \\
\hline VSsrna145 & VSAL_I2441/VSAL_I2442 & 2615332 & 2615451 & 120 & $>>>$ & +++ & - & - & Novel \\
\hline VSsrna152 & VSAL_I2535/VSAL_I2536 & 2717523 & 2717659 & 137 & $>>>$ & ++ & Fur-box & + & Novel \\
\hline VSsrna165 & VSAL_I2652/VSAL_I2653 & 2872308 & 2871972 & 337 & $<<<$ & +++ & - & + & RNaseP_bact_a (rnpB) \\
\hline VSsrna185 & VSAL_I2907/VSAL_I2908 & 3152443 & 3152724 & 282 & $<><$ & +++ & Sigma70 & - & $\begin{array}{l}\text { B2 V. cholerae, } \\
\text { Livny et al. }[2005]\end{array}$ \\
\hline VSsrna190 & VSAL_I2972/VSAL_I2973 & 3219049 & 3219240 & 192 & $<><$ & +++ & Sigma70 & + & Novel \\
\hline VSAsrna8 & VSAL_II0231/VSAL_II0232 & 254998 & 255222 & 226 & $>><$ & +++ & SigmaS & + & Novel \\
\hline VSAsrna17 & VSAL_II0520/VSAL_II0521 & 580917 & 580759 & 160 & $<<<$ & + & - & - & Novel \\
\hline VSAsrna46 & VSAL_II0920/VSAL_II0921 & 1006466 & 1006637 & 173 & $>><$ & + & - & + & Novel \\
\hline
\end{tabular}

\footnotetext{
${ }^{1}$ Gene numbers for the up- and downstream A. salmonicida open reading frames.

${ }^{2,3}$ Predicted sRNA coordinates and length (nt).

${ }^{4}$ Genes encoded on plus strand are denoted with $>$, and genes encoded on the minus strand are denoted with $<$.

${ }^{5}$ Blastn was used to search for sequence conservation in other bacteria. $+=$ Sequence conservation mainly in Aliivibrio $;++=$ sequence conservation primarily in Vibrionaceae family; $+++=$ sequence was conserved in many bacterial species.

${ }^{6}$ Promoter prediction with BProm software and in-house positronspecific scoring matrices and using Patser software [Hertz and Stormo, 1999].

${ }^{7}$ Rho-independent terminator as predicted by TransTerm software [Ermolaeva et al., 2000].

${ }^{8}$ sRNAs were queried against Rfam database [Gardner et al., 2009] and other experimentally validated Vibrionaceae sRNAs. Homologs to known sRNAs are indicated.
}

with HapR indirectly activating expression of Qrr [Svenningsen et al., 2008]. It is unclear to us why the level of Qrr is significantly higher in the A. salmonicida LitR mutant, but one possible explanation is that the litR mRNA target is absent, and Qrr will therefore not be degraded with its target. It should be noted that the number of $q r r$ genes varies between members of Vibrionaceae, for example one in A. salmonicida and Vibrio fischeri, four in $V$. cholerae, and five in V. vulnificus and Vibrio harveyi [Miyashiro et al., 2010], which suggests species-specific regulation of $q r r$ expression.

Probes targeted to VSsrna7, VSsrna17, VSsrna108, VSsrna152, and VSAsrna8 each produced one distinct band in close agreement with the predicted size. Probes against VSsrna21, VSsrna145, and VSsrna190 produced, in contrast, bands corresponding to larger RNAs than predicted, which is not unexpected since prediction of promoters and Rho-independent terminators is relatively unreliable, even in the model organism E. coli. The VSAsrna46 probe produced three distinct bands with the strongest band corresponding to an RNA close to the predicted size.

Interestingly, the resulting data suggest that several sRNAs are differentially expressed. In the $\Delta l i t R$ mutant, VSsrna7, VSsrna108, VSAsrna8, and VSAsrna46 are all upregulated during late exponential phase. VSsrna21 is in contrast downregulated $\sim 100$-fold. The on/off-like expression pattern of VSsrna21 indicates that it might have a direct role in quorum sensing. It does not share significant sequence conservation with any known sRNAs but appears to be conserved in other bacterial species (table 2). Under low iron conditions, VSsrna7 is upregulated 2.4and 2.9-fold, 7.5 and 15 min after treatment, respectively, whereas VSsrna190 is downregulated after treatment compared to the wild type. Under oxidative stress, VSsrna7 and VSsrna108 are upregulated 2.1- and 2.5-fold, respectively, 15 min after stimulation. The two latter ncRNAs were not noted as differentially expressed using microarray, which demonstrates the importance of using other direct methods, like Northern blot analysis, for verification of results.

In summary, of the 28 microarray spots that were chosen for further investigation using Northern blot analysis, we convincingly identified 11 sRNAs from A. salmonicida, including 9 new sRNAs, the homolog of B2 from V. cholerae, and Qrr. Six of the new sRNAs are in close agreement with predicted sizes, and 3 are longer than predicted (fig. 3), 8 (VSsrna 7, 108, 145, 152, and 190 and VSAsrna 8,17 , and 46) produced spots with high intensities $(>500)$ in microarray analysis, whereas one (VSsrna21) produced lower fluorescence signal (i.e. 76). We have tested the majority of putative sRNAs that produced high-intensity spots in microarray analysis using 
Northern blot analysis, and we find it reasonable to believe that many of these, if not all, are genuine sRNAs. We expect, however, that many more sRNAs remain to be discovered in A. salmonicida, also among the predicted RNAs that were identified with microarray analysis, but that remains to be further tested.

\section{Comparison with Other ncRNA Studies from the \\ Family Vibrionaceae}

A few studies have during recent years reported the discovery of ncRNAs in related members of Vibrionaceae [Liu et al., 2009; Livny et al., 2005; Silveira et al., 2010], and we wanted to compare our findings with these results. Twenty-five of our predicted ncRNAs are homologs of known ncRNAs found in Rfam and are distributed among the classes riboswitch (10), RNaseP (1), tmRNA (1), sRNA (6), SRP-RNA (1), anti-sense RNA (1), t44 (1), group II intron (1), and mRNA leaders (3) (see online suppl. table S1 for details). Compared to results from $V$. cholera, our predictions include 14 of 27 previously verified or putative IGR ncRNAs, including 3 of 6 ncRNAs (sRNApred B2, B4 and $\mathrm{C} 1$ ) that were predicted by sRNAPredict and confirmed by Northern analysis [Livny et al., 2005] and 4 of the 7 IGR sRNAs that were found using direct cloning and parallel sequencing [Sharma and Vogel, 2009]. Of the 7 IGR RNAs from the latter study, our prediction did not include IGR1, IGR4, and IGR6. IGR1 might be present in A. salmonicida (Blastn E-value $=0.065$ and total score $=$ 32), whereas IGR4 and IGR6 are only found in V. cholerae.

Using RNA-seq, Liu et al. [2009] reported 500 IGR RNAs in $V$. cholerae that were considered as putative sRNAs. We removed from this list redundant/overlapping RNA sequences and compared the resulting 251 non-redundant IGR sequences (199 and 52 in chromosomes I and II, respectively) with our 252 predicted ncRNAs using Blastn and the search criteria E-value $\leq 0.01$ and total score $\geq 30$. This search identified 21 significant hits (see online suppl. table S1 for details). Nine hits represent homologs of previously known ncRNAs in Rfam (6 riboswitches and 3 sRNAs) and 12 represent novel sRNAs (4 of which have been confirmed with Northern blot analysis).

In a recent study, identification of ncRNAs was performed on environmental vibrios [Rutherford et al., 2000] using a combination of in silico search (Rfam) followed by microarray-based expression profiling. Microarray analysis revealed $21 \mathrm{ncRNAs}$ that were expressed in mid-exponential phase, and a subset of six ncRNAs was tested with reverse transcription PCR. Our predictions identified 17 of these 21 ncRNAs.

\section{Conclusions}

We have used computational and experimental methods to identify intergenic ncRNAs (mostly sRNAs) in the marine bacterium A. salmonicida, and this is the first genome-wide study of its kind for a fish pathogen. Of the 252 tested ncRNAs, our microarray analysis suggests that approximately $\sim 50$ (i.e. when using a conservative threshold value) are expressed under our laboratory growth conditions, and Northern blot analysis verified expression of 9 new sRNAs. Our prediction list and expression profiling data also include sRNA candidates recently found in $V$. cholerae with RNA-seq and other methods. One of our current goals is to study the presumably important roles of sRNAs in the development of cold-water vibriosis in Atlantic salmon (and other marine fish species) caused by A. salmonicida. By comparing our data with results from other representatives of Vibrionaceae, such as the infamous human pathogen $V$. cholerae, we are discovering sRNAs that are broadly distributed in Vibrionaceae, as well as sRNAs that are limited to aliivibrios and A. salmonicida in particular. These analyses will hopefully guide us to better understand the biological roles of sRNAs in this important group of pathogens.

\section{Acknowledgements}

This work was supported by University of Tromsø, the Norwegian Research Council and Research and Functional Genomics in Norway. We are grateful to Ruth H. Paulssen and The Microarray Resource Centre in Tromsø for offering facilities and equipment. We wish to thank Christopher G. Fenton for advice on microarray data analysis and Lotte Olsen and Anja Strauss for technical assistance.

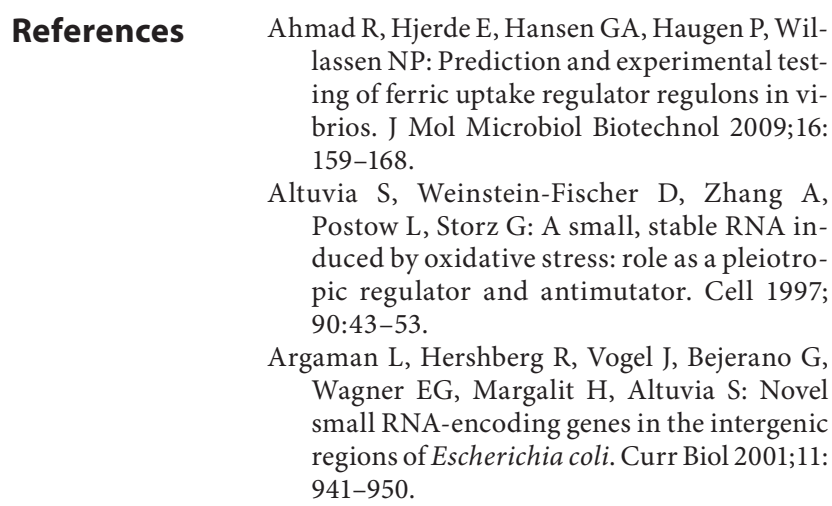

J Mol Microbiol Biotechnol 2012;22:352-360 
Bjelland AM, Sorum H, Tegegne DA, WintherLarsen HC, Willassen NP, Hansen H: LitR of Vibrio salmonicida is a salinity-sensitive quorum-sensing regulator of phenotypes involved in host interactions and virulence. Infect Immun 2012;80:1681-1689.

-Breaker RR: Prospects for riboswitch discovery and analysis. Mol Cell 2011;43:867-879.

-Chen S, Lesnik EA, Hall TA, Sampath R, Griffey RH, Ecker DJ, Blyn LB: A bioinformatics based approach to discover small RNA genes in the Escherichia coli genome. Biosystems 2002;65:157-177.

Davis BM, Quinones M, Pratt J, Ding Y, Waldor MK: Characterization of the small untranslated RNA RyhB and its regulon in Vibrio cholerae. J Bacteriol 2005; 187:4005-4014. Davis BM, Waldor MK: RNase E-dependent pro-
cessing stabilizes MicX, a Vibrio cholerae sRNA. Mol Microbiol 2007;65:373-385.

Dysvik B, Jonassen I: J-Express: exploring gene expression data using Java. Bioinformatics 2001;17:369-370.

-Ermolaeva MD, Khalak HG, White O, Smith HO, Salzberg SL: Prediction of transcription terminators in bacterial genomes. J Mol Biol 2000;301:27-33.

- Gardner PP, Daub J, Tate JG, Nawrocki EP, Kolbe DL, Lindgreen S, Wilkinson AC, Finn RD, Griffiths-Jones S, Eddy SR, Bateman A: Rfam: updates to the RNA families database. Nucleic Acids Res 2009;37:D136-D140.

Gottesman S: Micros for microbes: Non-coding regulatory RNAs in bacteria. Trends Genet 2005;21:399-404.

Griffin BE: Separation of 32P-labelled ribonucleic acid components. The use of polyethylenimine-cellulose (TLC) as a second dimension in separating oligoribonucleotides of '4.5 S' and 5 S from E. coli. FEBS Lett 1971;15: 165-168.

-Hammer BK, Bassler BL: Regulatory small RNAs circumvent the conventional quorum sensing pathway in pandemic Vibrio cholerae. Proc Natl Acad Sci USA 2007;104:11145-11149.

- Hansen GA, Ahmad R, Hjerde E, Fenton CG, Willassen NP, Haugen P: Expression profiling reveals spot 42 small RNA as a key regulator in the central metabolism of Alivivibrio salmonicida. BMC Genomics 2012;13:37.

-Hertz GZ, Stormo GD: Identifying DNA and protein patterns with statistically significant alignments of multiple sequences. Bioinformatics 1999; 15:563-577.

-Hjerde E, Lorentzen MS, Holden MT, Seeger K, Paulsen S, Bason N, Churcher C, Harris D, Norbertczak H, Quail MA, Sanders S, Thurston S, Parkhill J, Willassen NP, Thomson NR: The genome sequence of the fish pathogen Aliivibrio salmonicida strain LFI1238 shows extensive evidence of gene decay. BMC Genomics 2008;9:616.

-Horvath P, Barrangou R: Crispr/cas, the immune system of bacteria and archaea. Science 2010; 327:167-170.

-Huang HY, Chang HY, Chou CH, Tseng CP, Ho SY, Yang CD, Ju YW, Huang HD: sRNAMap: genomic maps for small non-coding RNAs, their regulators and their targets in microbial genomes. Nucleic Acids Res 2009; 37:D150-D154

-Ikemura T, Dahlberg JE: Small ribonucleic acids of Escherichia coli. I. Characterization by polyacrylamide gel electrophoresis and fingerprint analysis. J Biol Chem 1973;248: 5024-5032.

Irnov I, Sharma CM, Vogel J, Winkler WC: Identification of regulatory RNAs in Bacillus subtilis. Nucleic Acids Res 2010;38:6637-6651.

Le Roux F, Davis BM, Waldor MK: Conserved small RNAs govern replication and incompatibility of a diverse new plasmid family from marine bacteria. Nucleic Acids Res 2011;39:1004-1013.

Lenz DH, Miller MB, Zhu J, Kulkarni RV, Bassler BL: CsrA and three redundant small RNAs regulate quorum sensing in Vibrio cholerae. Mol Microbiol 2005;58:1186-1202.

Lenz DH, Mok KC, Lilley BN, Kulkarni RV, Wingreen NS, Bassler BL: The small RNA chaperone $\mathrm{Hfq}$ and multiple small RNAs control quorum sensing in Vibrio harveyi and Vibrio cholerae. Cell 2004;118:69-82.

-Liu JM, Livny J, Lawrence MS, Kimball MD, Waldor MK, Camilli A: Experimental discovery of sRNAs in Vibrio cholerae by direct cloning, 5s/tRNA depletion and parallel sequencing. Nucleic Acids Res 2009;37:e46.

Livny J, Brencic A, Lory S, Waldor MK: Identification of 17 Pseudomonas aeruginosa sRNAs and prediction of sRNA-encoding genes in 10 diverse pathogens using the bioinformatic tool sRNAPredict2. Nucleic Acids Res 2006;34:3484-3493.

Livny J, Fogel MA, Davis BM, Waldor MK: sRNAPredict: an integrative computational approach to identify sRNAs in bacterial genomes. Nucleic Acids Res 2005;33:40964105.

-Masse E, Gottesman S: A small RNA regulates the expression of genes involved in iron metabolism in Escherichia coli. Proc Natl Acad Sci USA 2002;99:4620-4625.

Milton DL: Quorum sensing in vibrios: complexity for diversification. Int J Med Microbiol 2006;296:61-71.

-Miyashiro T, Wollenberg MS, Cao X, Oehlert D, Ruby EG: A single qrr gene is necessary and sufficient for LuxO-mediated regulation in Vibrio fischeri. Mol Microbiol 2010;77:15561567.

Mustachio LM, Aksit S, Mistry RH, Scheffler R, Yamada A, Liu JM: The Vibrio cholerae mannitol transporter is regulated posttranscriptionally by the MtlS small regulatory RNA. J Bacteriol 2012;194:598-606.

Nawrocki EP, Kolbe DL, Eddy SR: Infernal 1.0: inference of RNA alignments. Bioinformatics 2009;25:1335-1337.

Ortega A, Gonzalo-Asensio J, Garcia-Del Portillo F: Dynamics of Salmonella small RNA expression in non-growing bacteria located inside eukaryotic cells. RNA Biol 2012;9: 469-488.
Passalacqua KD, Varadarajan A, Ondov BD, Okou DT, Zwick ME, Bergman NH: Structure and complexity of a bacterial transcriptome. J Bacteriol 2009;191:3203-3211.

-Pedersen HL, Hjerde E, Paulsen SM, Hansen H, Olsen L, Thode SK, Santos MT, Paulssen RH, Willassen NP, Haugen P: Global responses of Alivibrio salmonicida to hydrogen peroxide as revealed by microarray analysis. Mar Genomics 2010;3:193-200.

Pichon C, Felden B: Small RNA genes expressed from Staphylococcus aureus genomic and pathogenicity islands with specific expression among pathogenic strains. Proc Natl Acad Sci USA 2005;102:14249-14254.

Rivas E, Eddy SR: Noncoding RNA gene detection using comparative sequence analysis. BMC Bioinformatics 2001;2:8.

Romby P, Vandenesch F, Wagner EG: The role of RNAs in the regulation of virulence-gene expression. Curr Opin Microbiol 2006;9:229236.

Rutherford K, Parkhill J, Crook J, Horsnell T, Rice P, Rajandream MA, Barrell B: Artemis: sequence visualization and annotation. Bioinformatics 2000;16:944-945.

Sharma CM, Vogel J: Experimental approaches for the discovery and characterization of regulatory small RNA. Curr Opin Microbiol 2009; 12:536-546.

-Silveira AC, Robertson KL, Lin B, Wang Z, Vora GJ, Vasconcelos AT, Thompson FL: Identification of non-coding RNAs in environmental vibrios. Microbiology 2010;156:24522458.

-Song T, Mika F, Lindmark B, Liu Z, Schild S, Bishop A, Zhu J, Camilli A, Johansson J, Vogel J, Wai SN: A new Vibrio cholerae sRNA modulates colonization and affects release of outer membrane vesicles. Mol Microbiol 2008;70:100-111.

-Svenningsen SL, Waters CM, Bassler BL: A negative feedback loop involving small RNAs accelerates Vibrio cholerae's transition out of quorum-sensing mode. Genes Dev 2008;22: 226-238.

- Thompson JR, Randa MA, Marcelino LA, Tomita-Mitchell A, Lim E, Polz MF: Diversity and dynamics of a North Atlantic coastal vibrio community. Appl Environ Microbiol 2004; 70:4103-4110.

Tjaden B: TargetRNA: A tool for predicting targets of small RNA action in bacteria. Nucleic Acids Res 2008;36:W109-W113.

Vanderpool CK: Physiological consequences of small RNA-mediated regulation of glucosephosphate stress. Curr Opin Microbiol 2007; 10:146-151.

-Wassarman KM, Repoila F, Rosenow C, Storz G Gottesman S: Identification of novel small RNAs using comparative genomics and microarrays. Genes Dev 2001;15:1637-1651.

Wassarman KM, Zhang A, Storz G: Small RNAs in Escherichia coli. Trends Microbiol 1999;7: 37-45.

Westhof E: The amazing world of bacterial structured RNAs. Genome Biol 2010;11:108. 